\title{
EXPLICIT FUNCTIONAL DETERMINANTS IN FOUR DIMENSIONS
}

\author{
THOMAS P. BRANSON AND BENT ØRSTED
}

(Communicated by Palle E. T. Jorgensen)

\begin{abstract}
Working on the four-sphere $S^{4}$, a flat four-torus, $S^{2} \times S^{2}$, or a compact hyperbolic space, with a metric which is an arbitrary positive function times the standard one, we give explicit formulas for the functional determinants of the conformal Laplacian (Yamabe operator) and the square of the Dirac operator, and discuss qualitative features of the resulting variational problems. Our analysis actually applies in the conformal class of any Riemannian, locally symmetric, Einstein metric on a compact 4-manifold; and to any geometric differential operator which has positive definite leading symbol, and is a positive integral power of a conformally covariant operator.
\end{abstract}

\section{INTRODUCTION. Functional DETERMINANTS IN TWO DIMENSIONS}

Let $(M, g)$ be an $n$-dimensional compact manifold without boundary, and let $A$ be a formally selfadjoint, geometric partial differential operator with positive definite leading symbol. The order of $A$ is then necessarily a positive even integer $2 \ell$. We further assume that $A$ scales as its leading term does: if $\bar{g}=c^{2} g, 0<c \in \mathbb{R}$, then $\bar{A}=c^{-2 \ell} A$. For example, $A$ could be the Laplacian on some tensor bundle, or, if $M$ has spin structure, the square of the Dirac operator.

Heat equation theory (see, e.g., [Se, G1, G3]) tells us that the trace of the heat operator $\exp (-t A), t>0$, has a small-time asymptotic expansion

$$
\operatorname{Tr}_{L^{2}} \exp (-t A) \sim \sum_{i=0}^{\infty} t^{(2 i-n) / 2 \ell} a_{i}[A], \quad t \downarrow 0,
$$

in which the coefficients $a_{i}[A]$ are integrals of universal local expressions $U_{i}[A]$. (Addition of universal exact divergences to the $U_{i}$ does not change the $a_{i}$, but the $U_{i}$ are fixed by the requirement that they appear in the pointwise asymptotic expansion of the fiberwise trace of the heat kernel.)

Received by the editors April 9, 1990 and, in revised form May 5, 1990.

1980 Mathematics Subject Classification (1985 Revision). Primary 47F05.

Both authors were partially supported by NATO Collaborative Research Grant $720 / 84$. Thomas P. Branson was partially supported by NSF (U.S.) grant DMS-8696098, by the Danish Research Academy, and by a University of Iowa Faculty Scholar Award. He gratefully acknowledges the hospitality and services of University House at the University of Iowa. 
Our analytic assumptions guarantee that $A$ will have pure real eigenvalue spectrum $\left\{\lambda_{j} \mid j \in \mathbb{N}\right\}$ bounded below ( $\mathbb{N}$ denoting the natural numbers), and in fact that $\lambda_{j} \sim$ const $\cdot j^{2 \ell / n}$ as $j \uparrow \infty$. The zeta function $\zeta_{A}(s)$ is defined for large $\operatorname{Re} s$ by

$$
\zeta_{A}(s)=\sum_{\lambda_{j} \neq 0}\left|\lambda_{j}\right|^{-s},
$$

and analytic continuation gives a meromorphic extension with isolated simple poles. $\zeta_{A}(s)$ is regular at the nonpositive integers; in particular, $\zeta_{A}(0)+q[A]=$ $a_{n / 2}[A]$, where $q[A]$ is the number of zero modes, and $a_{n / 2}[A]$ is taken to be zero when $n$ is odd. The functional determinant of $A$ is defined by

$$
\log |\operatorname{det} A|=-\zeta_{A}^{\prime}(0), \quad \text { sign det } A=(-1)^{\#\left\{\lambda_{j}<0\right\}} .
$$

Note that the determinant is not scale-invariant:

$$
\bar{g}=c^{2} g, 0<c \in \mathbb{R} \Rightarrow \operatorname{det} \bar{A}=c^{-2 \ell \zeta_{A}(0)} \operatorname{det} A .
$$

But this says exactly that the functional

$$
p_{A}(g)=\left(\frac{\operatorname{vol} g}{v_{0}}\right)^{2 \ell \zeta_{A}(0) / n} \operatorname{det} A,
$$

where $v_{0}$ is a positive constant, is scale-invariant. (Note that $\zeta_{A}(0)$ is also scale-invariant.) As an alternative to looking at the functional $p_{A}$, we could freeze out scale changes by demanding that all our manifolds have volume $v_{0}$.

A conformally covariant operator is a geometric differential operator $A$ for which pointwise scaling of the metric $\bar{g}=e^{2 \omega} g, \omega$ a smooth function, produces a change

$$
\bar{A}=e^{-b \omega} A e^{a \omega}
$$

in the operator, for some constants $a$ and $b$. (The $e^{a \omega}$ on the right is to be interpreted as a multiplication operator.) Under our scaling assumptions, $b$ is necessarily $a+2 \ell$. Conformal covariance can be rephrased in terms of conformal invariance of an operator between density bundles, but we shall refrain from doing so here. For a conformally covariant operator $A$ satisfying our analytic assumptions, $a_{n / 2}[A], q[A]$, and thus their difference $\zeta_{A}(0)$, as well as $\#\left\{\lambda_{j}<0\right\}$ are conformal invariants $[\mathrm{BØ} 1] ; \zeta_{A}(0)$ is sometimes called the trace anomaly of $A$. The conformal variation of $\operatorname{det} A$ is given by a local formula [BØ3, Theorem 3.7]:

$$
\left.(d / d u)\right|_{u=0} \zeta_{A}^{\prime}(0)=2 \ell \int_{M} \omega\left(U_{n / 2}[A]-\sum_{\lambda_{j}=0}\left|\varphi_{j}\right|^{2}\right) \mathrm{dvol} .
$$

Here we allow the metric to run through the conformal curve $e^{2 u \omega} g$, and the $\varphi_{j}$ are an orthonormal basis of the zero eigenspace in the background metric $g$. In addition, we have the same formulas for the conformal variations of $\zeta_{A}(0)$ and $\zeta_{A}^{\prime}(0)$ even when $A$ is only a positive integral power of a conformal 
covariant $B$ [BØ2, $\mathrm{B} \varnothing 3]$ ( $A$ need not be conformally covariant and $B$ need not have positive definite leading symbol), the prototypical example being the square of the Dirac operator. Note that (1.2) can also be written

$$
\left.(d / d u)\right|_{u=0}\left(-\log p_{A}(g)\right)=2 \ell v_{0}\left(\left(\omega W_{n / 2}\right)^{\sim}-\widetilde{\omega} \widetilde{W}_{n / 2}\right),
$$

where $W_{n / 2}=U_{n / 2}-\Sigma_{\lambda_{j}=0}\left|\varphi_{j}\right|^{2}$, and a tilde denotes the average over $(M, \mathrm{dvol})$. In particular, metrics $\bar{g}=e^{2 \omega} g$ critical for $p_{A}(g)$ when $\omega$ is restricted by $\int \omega=0$ must have constant $\bar{W}_{n / 2}$.

The situation in dimension two has been considered by many authors [Poly12, Polc, We1-2, OPS]. Here the Laplacian $\Delta=\delta d=-\nabla^{i} \nabla_{i}$ is conformally covariant, and $U_{n / 2}[\Delta]=U_{1}[\Delta]=K / 24 \pi$, where $K=R^{i j}{ }_{i j}$ is the scalar curvature. The Laplacian has one zero mode, the constant functions, and thus

$$
\zeta_{\Delta}(0)=\int_{M} \frac{K}{24 \pi} \mathrm{dvol}-1=\frac{1}{6} \chi(M)-1,
$$

where $\chi(M)$ is the Euler characteristic. (1.2) in this case gives

$$
\left.(d / d u)\right|_{u=0} \zeta_{\Delta}^{\prime}(0)=2 \int_{M} \omega\left(U_{1}[\Delta]-v^{-1}\right) \mathrm{dvol},
$$

where $v$ is the area of $g$. Now a conformal change $\bar{g}=e^{2 \omega} g$ has the effects

$$
\bar{K}=e^{-2 \omega}(K+2 \Delta \omega)
$$

on the scalar curvature, and $\overline{\mathrm{dvol}}=e^{2 \omega} \mathrm{dvol}$ on the area element, so that (1.2) gives

$$
\frac{d}{d u} \zeta_{\Delta_{u}}^{\prime}(0)=\frac{1}{12 \pi} \int_{M} \omega(K+2 u \Delta \omega) \mathrm{dvol}-\frac{d\left(\log v\left(e^{2 u \omega} g\right)\right)}{d u}
$$

as $\bar{g}$ runs through the $e^{2 u \omega} g$. Integrating to $u=1$, we get

$$
\overline{\zeta_{\Delta}^{\prime}(0)}=\zeta_{\Delta}^{\prime}(0)=\zeta_{\Delta}^{\prime}(0)+\frac{1}{12 \pi} \int_{M} \omega(K+\Delta \omega) \mathrm{dvol}-\log \frac{v\left(e^{2 \omega} g\right)}{v(g)} .
$$

Concentrating on the case of the two-sphere $M=S^{2}$, if $g$ is the standard uniform metric (of scalar curvature 2 and area $4 \pi$ ), then $\Delta$ has eigenvalues $\lambda_{j}=j(j+1)$ with multiplicities $2 j+1$. A long calculation with these data gives $\zeta_{\Delta}(0)=-\frac{2}{3}$, and $\zeta_{\Delta}^{\prime}(0)=4 \zeta_{R}^{\prime}(-1)-\frac{1}{2}$, where $\zeta_{R}(s)$ is the classical Riemann zeta function. (See [We1, Appendix C]. In the notation of $\S 4$.e below, $\zeta_{\Delta}(s)=f_{1}(s)$.) Thus in this case,

$$
\overline{\zeta_{\Delta}^{\prime}(0)}=4 \zeta_{R}^{\prime}(-1)-\frac{1}{2}+\frac{1}{12 \pi}\left(2 \int_{S^{2}} \omega+\int_{S^{2}}|d \omega|^{2}\right)-\log \frac{v\left(e^{2 \omega} g\right)}{4 \pi} .
$$

The extremal problem that makes sense in this context is to maximize the determinant within the conformal class of the standard metric, subject to the 
area-preservation constraint $\int e^{2 \omega}=4 \pi$. Equivalently, we could maximize the functional

$$
p(\omega)=p_{\Delta}\left(e^{2 \omega} g\right)=\left(\frac{\int e^{2 \omega}}{4 \pi}\right)^{-2 / 3} \operatorname{det} \bar{\Delta},
$$

taking as our constraint any rule which distinguishes one member of each translation class $\{\omega+a \mid a \in \mathbb{R}\}$; most convenient is the linear constraint $\int \omega=0$. If $\omega$ solves this last problem, its translate

$$
\omega-\frac{1}{2} \log \frac{\int e^{2 \omega}}{4 \pi}
$$

solves the original problem. Thus our problem is to minimize

$$
\frac{1}{12 \pi} \int|d \omega|^{2}-\frac{1}{3} \log \int e^{2 \omega}
$$

subject to $\int \omega=0$. A delicate analysis [OPS, $\S 2.3$ ] shows that this functional is bounded below; by the remark following (1.3), minima can occur only at constant scalar curvature metrics. The uniformization theorem now guarantees that the minimum is attained exactly when $\bar{g}$ is (up to multiplication by a positive constant) obtained by a Möbius transformation (conformal diffeomorphism) from the standard metric $g$. Thus the maximum value of the determinant is its value at $\omega=0$, that being

$$
e^{1 / 2-4 \zeta_{R}^{\prime}(-1)}=3.19531 \ldots
$$

\section{FUNCTIONAL DETERMINANTS IN FOUR DIMENSIONS}

Suppose $A$ is a differential operator in dimension $n$ satisfying our analytic assumptions, and that $A$ is insensitive to changes in orientation. By Weyl's invariant theory, $U_{2}[A]$, and in fact every local scalar $O(n)$-invariant of its homogeneity, is a linear combination of $|C|^{2},|B|^{2}, J^{2}$, and $\Delta J$, where $J=$ $K / 2(n-1), B$ is the trace-free Ricci tensor $(r-K g / n) /(n-2), C$ is the Weyl conformal curvature tensor, $|B|^{2}=B^{i j} B_{i j}$, and similarly for $|C|^{2} . J$, $B$, and $C$ are the projections of the Riemann tensor onto the $O(n)$-irreducible summands of the vector bundle of algebraic curvature tensors. The orientationinsensivity assumption is unnecessary except in dimension four, where (on an oriented manifold) $C$ breaks up under $S O(4)$ into self-dual and anti-self-dual parts $C_{ \pm}$. This adds a new invariant, $\left|C_{+}\right|^{2}-\left|C_{-}\right|^{2}$, to our basis. This extra invariant shows up, for example, in the index theory of the signature and spin complexes, whose Laplacians are orientation-sensitive. Locally conformally flat metrics have $C=0$; Einstein metrics have $B=0$; by the second Bianchi identity, Einstein metrics in dimension greater than two have constant scalar curvature. A metric is locally symmetric if $\nabla J, \nabla B$, and $\nabla C$ vanish, and flat if $J, B$, and $C$ vanish. We shall use $V=B+J g / n$ to simplify the appearance of some formulas. 
If $A$ is a positive integral power of a conformal covariant, there is a relation among the coefficients of the four basic local invariants in the expression for $U_{2}[A][\mathrm{B} \varnothing 2, \S 5]$; in dimension four, this relation says that $U_{2}[A]$ is of the form

$$
\alpha_{1}|C|^{2}+\alpha_{2}\left(|B|^{2}-\frac{3}{4} J^{2}\right)+\alpha_{3} \Delta J .
$$

The four-dimensional Pfaffian (Gauss-Bonnet integrand)

$$
\mathrm{Pff}=\frac{1}{32 \pi^{2}}\left(|C|^{2}-8|B|^{2}+6 J^{2}\right)
$$

is also of the form (2.1), so $U_{2}[A]$ is a linear combination of $|C|^{2}$, Pff, and $\Delta J$.

There is a fourth-order operator which turns out to be very natural in this setting, playing some of the roles in dimension four that the Laplacian plays in dimension two. Paneitz' operator, originally introduced in connection with the interaction between the gauge and conformal groups for Maxwell's equations, [Pa, B2, ES, B3] is $P=P_{0}+(n-4) Q$, where $P_{0}$ is the operator

$$
P_{0}=\Delta^{2}+\delta\{(n-2) J-4 V \cdot\} d
$$

on functions ( $V \cdot$ is the natural action of a two-tensor on one-forms), and $Q$ is the quantity

$$
Q=\left(-4|V|^{2}+n J^{2}+2 \Delta J\right) / 4 \text {. }
$$

In fact, $P$ is conformally covariant:

$$
\bar{g}=e^{2 \omega} g \Rightarrow \bar{P}=e^{-(n+4) \omega / 2} P e^{(n-4) \omega / 2} .
$$

Applying this conformal covariance relation to the function 1 and taking advantage of the fact that $P_{0}$ annihilates constants, we get

$$
(n-4) \bar{Q}=e^{-(n+4) \omega / 2} P_{0}\left(e^{(n-4) \omega / 2}-1\right)+(n-4) e^{-4 \omega} Q .
$$

Analytically continuing in $n$ and dividing by $n-4$, we get

$$
\bar{Q}=e^{-4 \omega}\left(Q+\frac{1}{2} P_{0} \omega\right), \quad n=4 .
$$

(Note the similarity to (1.4) in dimension two.) Of course, it is hard to justify analytic continuation in the dimension, but explicit calculation with the local invariants involved also leads to $(2.3)$. Since $Q=\left(-4|B|^{2}+3 J^{2}+2 \Delta J\right) / 4$, like the Pfaffian, is of the form (2.1) in dimension four,

$$
U_{2}[A]=\beta_{1}|C|^{2}+\beta_{2} Q+\beta_{3} \Delta J
$$

for some constants $\beta_{m}$, provided $A$ is a positive integral power of a conformal covariant. Since $\overline{|C|^{2}}=e^{-4 \omega}|C|^{2}$, the only additional information we need is a formula for $\overline{\Delta J}$. Working just in dimension four,

$$
\bar{J}=e^{-2 \omega}\left(J+\Delta \omega-|d \omega|^{2}\right),
$$


and $\delta$ satisfies the conformal covariance relation $\bar{\delta}=e^{-4 \omega} \delta e^{2 \omega}$, so that

$$
\begin{aligned}
\overline{\Delta J}=\bar{\delta} d \bar{J}= & e^{-4 \omega}\left(\Delta J+\Delta^{2} \omega-2 J \Delta \omega+2\langle d J, d \omega\rangle\right. \\
& -\Delta|d \omega|^{2}-2(\Delta \omega)^{2}+2\langle d \omega, d \Delta \omega\rangle \\
& \left.+2|d \omega|^{2} \Delta \omega-2\left\langle d \omega, d|d \omega|^{2}\right\rangle\right), \quad n=4 .
\end{aligned}
$$

Thus for conformal, orientation-insensitive $A$ in dimension four,

$$
\overline{U_{2}[A]}=e^{-4 \omega}\left(U_{2}[A]+\frac{1}{2} \beta_{2} P_{0} \omega+\beta_{3}\left\{b_{1}(\omega)+b_{2}(\omega)+b_{3}(\omega)\right\}\right),
$$

where $b_{i}(\omega)$ is $i$-homogeneous in $\omega$ :

$$
\begin{aligned}
& b_{1}(\omega)=\Delta^{2} \omega-2 J \Delta \omega+2\langle d J, d \omega\rangle, \\
& b_{2}(\omega)=-\Delta|d \omega|^{2}-2(\Delta \omega)^{2}+2\langle d \omega, d \Delta \omega\rangle, \\
& b_{3}(\omega)=2|d \omega|^{2} \Delta \omega-2\left\langle d \omega, d|d \omega|^{2}\right\rangle .
\end{aligned}
$$

Now allow $g$ to run through the conformal curve $g_{u}=e^{2 u \omega} g$, and assume for the time being that $A$ has no zero modes. Then (1.2), together with $\mathrm{dvol}_{u}=$ $e^{4 u \omega} \mathrm{dvol}$, gives

$$
\begin{aligned}
\frac{d}{d u} \zeta_{A_{u}}^{\prime}(0)=2 \ell \int_{M} \omega( & U_{2}[A]+\frac{1}{2} \beta_{2} u P_{0} \omega \\
& \left.+\beta_{3}\left\{u b_{1}(\omega)+u^{2} b_{2}(\omega)+u^{3} b_{3}(\omega)\right\}\right) \mathrm{dvol},
\end{aligned}
$$

where $\mathrm{dvol}=\mathrm{dvol}_{0}$. Integrating to $u=1$, we get

$$
\begin{aligned}
\overline{\zeta_{A}^{\prime}(0)}= & \zeta_{A}^{\prime}(0) \\
& +2 \ell \int_{M} \omega\left(U_{2}[A]+\frac{1}{4} \beta_{2} P_{0} \omega+\beta_{3}\left\{\frac{1}{2} b_{1}(\omega)+\frac{1}{3} b_{2}(\omega)+\frac{1}{4} b_{3}(\omega)\right\}\right) \mathrm{dvol} .
\end{aligned}
$$

The functional $p_{A}$ (with $\left.v_{0}=v(g)\right)$ can now be written

$$
\begin{aligned}
-\log \left|p_{A}\right|\left(e^{2 \omega} g\right)+\log \left|p_{A}\right|(g) & \\
= & 2 \ell \int \omega\left(U_{2}[A]+\frac{1}{4} \beta_{2} P_{0} \omega+\beta_{3}\left\{\frac{1}{2} b_{1}(\omega)+\frac{1}{3} b_{2}(\omega)+\frac{1}{4} b_{3}(\omega)\right\}\right) \\
& -\frac{1}{2} \ell\left\{4 \pi^{2} \beta_{2} \chi(M)+\left(\beta_{1}-\frac{1}{8} \beta_{2}\right) \int|C|^{2}\right\} \log \frac{\int e^{4 \omega}}{v_{0}},
\end{aligned}
$$

using the fact that, in the absence of zero modes,

$$
\zeta_{A}(0)=\int U_{2}[A]=4 \pi^{2} \beta_{2} \chi(M)+\left(\beta_{1}-\frac{1}{8} \beta_{2}\right) \int|C|^{2} .
$$

Let us now specialize to the case in which $g$ has constant scalar curvature. (Schoen's proof of the Yamabe conjecture [Sc] shows that this is no loss of 
generality.) There are many ways of writing the integral involving the $b_{i}(\omega)$ in (2.8); one which we believe will be useful is obtained by integration by parts:

$$
\begin{aligned}
\int \omega\left\{\frac{1}{2} b_{1}(\omega)+\frac{1}{3} b_{2}(\omega)+\frac{1}{4} b_{3}(\omega)\right\} & =\frac{1}{2} \int\left(\Delta \omega-|d \omega|^{2}\right)^{2}-J \int|d \omega|^{2} \\
& =\frac{1}{2} \int\left(\frac{\Delta e^{\omega}}{e^{\omega}}\right)^{2}-J \int|d \omega|^{2} .
\end{aligned}
$$

If we make the stronger assumption that $g$ is locally symmetric, then $|C|^{2}$ and $U_{2}[A]$ will be constants related by

$$
U_{2}[A]=\left(\beta_{1}-\frac{1}{8} \beta_{2}\right)|C|^{2}+\frac{4 \pi^{2} \beta_{2} \chi(M)}{v_{0}} .
$$

If $g$ is Einstein, then $P_{0}=\Delta^{2}+J \Delta$. Thus we have:

Proposition 2.1. Let $(M, g)$ be a compact, locally symmetric, Einstein fourmanifold, and let $\omega \in C^{\infty}(M)$. Suppose $A$ is a formally selfadjoint positive power of a conformally covariant operator, that $A$ is orientation-insensitive, that $A$ has positive definite leading symbol, and that $A$ has no zero spectrum on $(M, g)$. Let $6 J$ and $c^{2}$ denote, respectively, the constant values of the scalar curvature and the norm-squared of the Weyl tensor. Then the functional $p_{A}$ at $\bar{g}=e^{2 \omega} g$ is related to that at $g$ by $\operatorname{sign} p_{A}(\bar{g})=\operatorname{sign} p_{A}(g)$,

$$
\begin{aligned}
\left(-\log \left|p_{A}\right|(\bar{g})+\log \left|p_{A}\right|(g)\right) / 2 \ell & \\
= & {\left[4 \pi^{2} \beta_{2} \chi(M)+\left(\beta_{1}-\frac{1}{8} \beta_{2}\right) v_{0} c^{2}\right]\left\{\frac{\int \omega}{v_{0}}-\frac{1}{4} \log \frac{\int e^{4 \omega}}{v_{0}}\right\} } \\
& +\frac{1}{4} \beta_{2} \int(\Delta \omega)^{2}+\left(\frac{1}{4} \beta_{2}-\beta_{3}\right) J \int|d \omega|^{2}+\frac{1}{2} \beta_{3} \int\left(\frac{\Delta e^{\omega}}{e^{\omega}}\right)^{2},
\end{aligned}
$$

where all integrals are taken with respect to the original metric $g$, and $U_{2}[A]=$ $\beta_{1}|C|^{2}+\beta_{2} Q+\beta_{3} \Delta J$ on general four-manifolds, $Q=\left(-4|B|^{2}+3 J^{2}+2 \Delta J\right) / 4$. The functional determinant of $A$ at $\bar{g}$ is related to that at $g$ by $\operatorname{sign} \operatorname{det} \bar{A}=$ sign $\operatorname{det} A$,

$$
\begin{aligned}
(-\log |\operatorname{det} \bar{A}|+\log |\operatorname{det} A|) / 2 \ell & \\
= & {\left[4 \pi^{2} \beta_{2} \chi(M)+\left(\beta_{1}-\frac{1}{8} \beta_{2}\right) v_{0} c^{2}\right] \frac{\int \omega}{v_{0}} } \\
& +\frac{1}{4} \beta_{2} \int(\Delta \omega)^{2}+\left(\frac{1}{4} \beta_{2}-\beta_{3}\right) J \int|d \omega|^{2}+\frac{1}{2} \beta_{3} \int\left(\frac{\Delta e^{\omega}}{e^{\omega}}\right)^{2} .
\end{aligned}
$$

Remark 2.2. The effect of zero modes on our formulas is as follows. Let $g_{u}=$ $e^{2 u \omega} g$, and let $\left\{\varphi_{j, u}\right\}$ be an $L^{2}\left(e^{4 u \omega} \mathrm{dvol}\right)$-orthonormal basis of the null space $\mathscr{N}\left(A_{u}\right)$. Then $\Phi_{u}^{2}(x)=\sum\left|\varphi_{j, u}(x)\right|_{u}^{2}$ is independent of our choices, and is 
jointly smooth in $x$ and $u$. We get an extra term $-2 \ell \int_{M} \omega \Phi_{u}^{2} e^{4 u \omega}$ dvol on the right in (2.6), leading to an extra $-2 \ell \Psi_{1}(\omega) \equiv-2 \ell \int_{M} \omega\left\{\int_{0}^{1} \Phi_{u}^{2} e^{4 u \omega} d u\right\}$ dvol on the right in (2.7). (2.9) becomes

$$
\zeta_{A}(0)=-q[A]+\int U_{2}[A]=-q[A]+4 \pi^{2} \beta_{2} \chi(M)+\left(\beta_{1}-\frac{1}{8} \beta_{2}\right) \int|C|^{2},
$$

so that if $\Psi_{2}(\omega)=\frac{1}{4} q[A] \log \left(\int e^{4 \omega} / v_{0}\right)$, there is an extra $2 \ell\left(\Psi_{2}(\omega)-\Psi_{1}(\omega)\right)$ on the right in (2.8). $\Psi_{2}-\Psi_{1}$ should be added on the right in the first formula of Proposition $2.1,-\Psi_{1}$ in the second. This seems fairly unsatisfying, as $\Psi_{1}$ still involves the whole conformal curve of metrics. But in many important applications, we have explicit enough knowledge of the null spaces of the $A_{u}$ that we can express $\Psi_{1}$ just in terms of the volumes of these metrics, and sometimes (as in the two-dimensional case) we can perform the remaining $u$ integration explicitly. (See $\S 3 . \mathrm{b}$ below.)

Suppose again that $\mathscr{N}(A)=0$. As in dimension two, we have the choice of extremizing the determinant subject to $\int e^{4 \omega}=v_{0}$, or extremizing $p_{A}(\omega)$ subject to $\int \omega=0$; if $\omega$ solves the latter problem,

$$
\omega-\frac{1}{4} \log \frac{\int e^{4 \omega}}{v_{0}}
$$

solves the former. Thus our problem is to extremize

$$
\begin{aligned}
F(\omega)= & \frac{1}{4} \beta_{2} \int(\Delta \omega)^{2}+\left(\frac{1}{4} \beta_{2}-\beta_{3}\right) J \int|d \omega|^{2}+\frac{1}{2} \beta_{3} \int\left(\frac{\Delta e^{\omega}}{e^{\omega}}\right)^{2} \\
& -\left[\pi^{2} \beta_{2} \chi(M)+\frac{1}{4}\left(\beta_{1}-\frac{1}{8} \beta_{2}\right) v_{0} c^{2}\right] \log \frac{\int e^{4 \omega}}{v_{0}}
\end{aligned}
$$

subject to $\int \omega=0$. A reasonable goal might be to prove that the determinant is extremized only at a uniform metric and its conformal transforms (if any). If we are to imitate the treatment of the Laplacian in two dimensions [OPS], we should use a convexity argument to prove that $F(\omega)$ is bounded from one side, and then prove that constant $U_{2}[A]$ (which we must have at critical metrics) implies uniformity. This last hurdle might be cleared just by tensor calculations as in Obata's theorem, which states that a constant scalar curvature metric in the conformal class of the standard metric $g$ on the sphere $S^{n}, n \geq 3$, must be obtained from $g$ by a conformal diffeomorphism. More likely, one would have to show that the gradient flow of $F(\omega)$ always leads to uniform metrics. The character of this problem will obviously depend heavily on the topology and geometry of $(M, g)$ (through $\chi(M), c^{2}$, and $J$ ), and on the operator $A$ (through the $\beta_{m}$ ). Not all of the topological/geometric data is independent, as $\chi(M)=\left(c^{2}+6 J^{2}\right) v_{0} / 32 \pi^{2}$. Note that by (2.2), a manifold supporting an Einstein metric $g$ in dimension four has $\chi(M) \geq 0$, with $\chi(M)=0$ if and 
only if $g$ is flat. There are many manifolds and operators to choose from; we discuss a few of these in the following sections.

\section{SPECIAL MANifOldS}

a. Let $M$ be the four-sphere $S^{4}$, and let $g$ be the standard metric (the pullback of the standard $\mathbb{R}^{5}$ metric to the unit sphere). Suppose that $\mathscr{N}(A)=0$. Our constants are $\chi(M)=2, c^{2}=0, J=2$, and $v_{0}=8 \pi^{2} / 3$, so that

$$
\begin{aligned}
F(\omega)= & \frac{1}{4} \beta_{2} \int(\Delta \omega)^{2}+\left(\frac{1}{2} \beta_{2}-2 \beta_{3}\right) \int|d \omega|^{2} \\
& +\frac{1}{2} \beta_{3} \int\left(\frac{\Delta e^{\omega}}{e^{\omega}}\right)^{2}-2 \pi^{2} \beta_{2} \log \frac{3 \int e^{4 \omega}}{8 \pi^{2}} .
\end{aligned}
$$

Thus $F(\omega)$ is never just the integral of a nonnegative quantity. Of course, $F(\omega)$ is obliged to be complicated (unless it vanishes identically) in the case of the four-sphere, since elements of the fifteen-dimensional group of conformal diffeomorphisms (like any diffeomorphisms) will carry a geometrically natural operator to an isospectral operator; thus the functional determinant at $e^{2 \omega} g$ will agree with that at $g$ when the conformal factor $\omega$ is implemented by a one-parameter group of conformal diffeomorphisms. (Note that such an $\omega$ need not satisfy the constraint $\int \omega=0$, so $F$ will only annihilate the translate $\left.\omega-\left(\int \omega\right) / v_{0}.\right)$ Thus there should be no isolated extremals.

On the other hand, we can show that any curve $e^{2 u \omega} g$ of extremal metrics with $\int \omega=0(u \in(-\varepsilon, \varepsilon), \varepsilon>0)$ must be implemented by a one-parameter group of conformal diffeomorphisms. Indeed, at an extremal, $U_{2}[A]=$ const, so for our extremal curve,

$$
\left.(d / d u)\right|_{u=0} U_{2}\left[A_{u}\right]=\text { const. }
$$

But the conformal invariance of the trace anomaly $\int U_{2}[A]$ shows that

$$
\left.(d / d u)\right|_{u=0} U_{2}\left[A_{u}\right]=-4 \omega U_{2}[A]+\text { divergence },
$$

so

By (2.5),

$$
\left.(d / d u)\right|_{u=0} U_{2}\left[A_{u}\right]=-12 U_{2}[A] \frac{\int \omega}{8 \pi^{2}}=0 .
$$

$$
\begin{aligned}
\left.(d / d u)\right|_{u=0} U_{2}\left[A_{u}\right] & =\left\{\left(\frac{1}{2} \beta_{2}+\beta_{3}\right) \Delta^{2}+\left(\beta_{2}-4 \beta_{3}\right) \Delta-12 \beta_{2}\right\} \omega \\
& =\left\{\left(\frac{1}{2} \beta_{2}+\beta_{3}\right) \Delta+3 \beta_{2}\right\}(\Delta-4) \omega .
\end{aligned}
$$

Thus $\omega$ must be in the 4-eigenspace of the Laplacian $\Delta$; that is, $\omega$ must be a first-order spherical harmonic, so long as $\beta_{2}$ and $\beta_{3}$ are not both zero, and the number $-6 \beta_{2} /\left(\beta_{2}+2 \beta_{3}\right)$ is not a higher eigenvalue of the Laplacian. (In the cases of the conformal Laplacian and the square of the Dirac operator, this number is -2 and $-11 / 3$ respectively; see $\S 4$ below.) But the first-order spherical harmonics (the span of the 5 homogeneous coordinate functions on $S^{4}$ ) generate the nonisometric conformal diffeomorphism directions. 
b. Let $(M, g)$ be a flat four-torus $\mathbb{R}^{4} / \Gamma$, where $\Gamma$ is a discrete subgroup of full rank, with the inherited metric. Here we expect to deal with zero modes, but very tractable ones. Our constants are $\chi(M)=c^{2}=J=0$, so that

$$
F(\omega)=\frac{1}{4} \beta_{2} \int(\Delta \omega)^{2}+\frac{1}{2} \beta_{3} \int\left(\frac{\Delta e^{\omega}}{e^{\omega}}\right)^{2} .
$$

In particular, replacing $\omega$ by $u \omega$ and letting $u \rightarrow \infty$, we see that $F(\omega)$ cannot be bounded below if $\beta_{3}<0$, or above if $\beta_{3}>0$.

Because of the presence of zero modes, what we really need to extremize (recalling Remark 2.2) is $F(\omega)+\Psi_{2}(\omega)-\Psi_{1}(\omega)$. Since $A$ is geometric, it is built polynomially from the covariant derivative and curvature, so for the flat metric, $A=A_{1} \nabla$ for some differential operator $A_{1}$. The vector bundle in which $A$ acts will be globally trivial, and in fact will admit a global orthogonal frame $\left\{\varphi_{j}\right\}$ of parallel sections of length $1 / \sqrt{v_{0}}$; these will be an $L^{2}$-orthonormal basis of $\mathscr{N}(A)$. Now introduce the conformal curve of metrics $g_{u}=e^{2 u \omega} g$. Our operator $A$ is a positive integral power of a conformal covariant $B$; say ord $B=r$, so that $A=B^{2 \ell / r}$. For some constant $a, B_{u}=e^{-(a+r) u \omega} B_{0} e^{a u \omega}$, so the $e^{-a u \omega} \varphi_{j}$ will be a $g_{u}$-orthogonal global frame. By [BØ3, Equation (4.1)], formal selfadjointness forces

$$
\left|e^{-a u \omega} \varphi_{j}\right|_{u}^{2} \mathrm{dvol}_{u}=e^{r u \omega}\left|\varphi_{j}\right|_{0}^{2} \mathrm{dvol} l_{0},
$$

so that the

$$
\varphi_{j, u}=\sqrt{\frac{v_{0}}{v_{r u / 4}}} e^{-a u \omega} \varphi_{j}
$$

are an $L^{2}\left(\mathrm{dvol}_{u}\right)$-orthonormal basis of $\mathscr{N}\left(A_{u}\right)$; in particular, $\Phi_{u}^{2}=q v_{r u / 4}^{-1} e^{(r-4) u \omega}$, where $q=q[A]$. Thus, performing the $u$-integration of Remark 2.2 explicitly, we get

$$
\Psi_{1}(\omega)=\frac{q}{r} \log \frac{v_{r / 4}}{v_{0}} .
$$

Recalling that $\Psi_{2}(\omega)=(q / 4) \log \left(v_{1} / v_{0}\right)$, we see that the correction

$$
\Psi_{2}(\omega)-\Psi_{1}(\omega)=q \log \frac{v_{1}^{1 / 4} v_{r / 4}^{-1 / r}}{v_{0}^{1 / r-1 / 4}}
$$

vanishes when $r=4$ (for example, if $A$ is Paneitz' operator). If $r<4$, the correction is easily estimated in terms of $v_{0}$ and $v_{1}$ : by the convexity of the exponential function, $v_{r / 4} \geq v_{1}^{r / 4}$, so

$$
q \log \frac{v_{1}^{1 / 4} \max \left(v_{0}, v_{1}\right)^{-1 / r}}{v_{0}^{1 / r-1 / 4}} \leq \Psi_{2}(\omega)-\Psi_{1}(\omega) \leq q \log v_{0}^{1 / 4-1 / r} .
$$

If $r=4$ and $\beta_{2} \beta_{3} \geq 0, F(\omega)$ is bounded on one side by zero, so the original flat metric will be an extremal. If $r=4, \beta_{2} \neq 0$ and $\beta_{2} \beta_{3} \geq 0$, the original metric will be the unique absolute extremal, since $\Delta \omega=0$ together with $\int \omega=0$ implies $\omega=0$. 
c. Let $(M, g)$ be $S^{2} \times S^{2}$ with the product of standard $S^{2}$ metrics. Then $\chi(M)=4, J=2 / 3$, and $v_{0}=16 \pi^{2}$, so that $c^{2}=16 / 3$ by $(2.2)$. (That this space is conformally curved is perhaps contrary to naive expectation; we remark that the standard pseudo-Riemannian metric of signature $(2,2)$ on $S^{2} \times S^{2}$ is conformally flat.) Our functional is

$$
\begin{aligned}
F(\omega)= & \frac{1}{4} \beta_{2} \int(\Delta \omega)^{2}+\left(\frac{1}{6} \beta_{2}-\frac{2}{3} \beta_{3}\right) \int|d \omega|^{2} \\
& +\frac{1}{2} \beta_{3} \int\left(\frac{\Delta e^{\omega}}{e^{\omega}}\right)^{2}-\frac{4 \pi^{2}}{3}\left[16 \beta_{1}+\beta_{2}\right] \log \frac{\int e^{4 \omega}}{16 \pi^{2}} .
\end{aligned}
$$

d. Let $(M, g)$ be a compact hyperbolic four-space $\Gamma \backslash \mathbb{H}^{4}, \Gamma$ a compact discontinuous group of isometries. Then $c^{2}=0$ and $J<0$. If we normalize the $\mathbb{H}^{4}$ metric so that $J=-2$ (i.e., so the sectional curvatures are -1 ), then $\chi(M)=3 v_{0} / 4 \pi^{2}$ by $(2.2)$; in particular, this number is a positive integer. If there are no zero modes, our functional is

$$
\begin{aligned}
F(\omega)=\frac{1}{4} & \beta_{2} \int(\Delta \omega)^{2}-2\left(\frac{1}{4} \beta_{2}-\beta_{3}\right) \int|d \omega|^{2} \\
& +\frac{1}{2} \beta_{3} \int\left(\frac{\Delta e^{\omega}}{e^{\omega}}\right)^{2}-\pi^{2} \beta_{2} \chi(M) \log \frac{3 \int e^{4 \omega}}{4 \pi^{2} \chi(M)} .
\end{aligned}
$$

Thus if $\beta_{2}=0 \neq \beta_{3}$, the original metric is the unique absolute extremal.

\section{SPECIAL OPERAtors. Remarks}

a. In $n$ dimensions, the conformal Laplacian $D=\Delta+(n-2) K / 4(n-1)=$ $\Delta+(n-2) J / 2$ on scalar functions is conformally covariant:

$$
\bar{g}=e^{2 \omega} g \Rightarrow \bar{D}=e^{-(n+2) \omega / 2} D e^{(n-2) \omega / 2} .
$$

In dimension 4,

$$
(4 \pi)^{2} \cdot 180 U_{2}[D]=|C|^{2}+4|B|^{2}-3 J^{2}-6 \Delta J
$$

by, e.g., [G1, p. 610$],[H]$, or $[\mathrm{B} \varnothing 1, \S 4 . b]$; that is,

$$
(4 \pi)^{2} \cdot 180\left(\beta_{1}, \beta_{2}, \beta_{3}\right)[D]=(1,-4,-4) \text {. }
$$

b. The square of the Dirac operator is also grist for our mill. Let $(M, g, \mathscr{O}, \gamma)$ be a compact, oriented spin manifold of dimension $n$. Here $\mathscr{O}$ is the orientation and $\gamma$ the fundamental section of $T \otimes \Sigma \otimes \Sigma^{*}$, where $T$ is the tangent and $\Sigma$ the spinor bundle. Let $\not \nabla=\gamma^{i} \nabla_{i}: C^{\infty}(M, \Sigma) \rightarrow C^{\infty}(M, \Sigma)$ be the Dirac operator. By, e.g., [K], $\not \nabla$ satisfies a conformal covariance relation

$$
\bar{g}=e^{2 \omega} g, \bar{\gamma}=e^{-\omega} \gamma \Rightarrow \bar{\nabla}=e^{-(n+1) \omega / 2} \not \nabla e^{(n-1) \omega / 2} .
$$

As noted in $\S 1, \not^{2}$ is not conformally covariant, but our machinery requires only a positive integral power of a conformal covariant. By [BØ2, pp. 98, 99], 
when $n=4$,

$$
\begin{aligned}
(4 \pi)^{2} \cdot 360 U_{2}\left[P^{2}\right] & =12 \Delta K+5 K^{2}-8|r|^{2}-7|R|^{2} \\
& =72 \Delta J+66 J^{2}-88|B|^{2}-7|C|^{2}
\end{aligned}
$$

Thus

$$
(4 \pi)^{2} \cdot 360\left(\beta_{1}, \beta_{2}, \beta_{3}\right)\left[P^{2}\right]=(-7,88,28) .
$$

c. For a general $A$ satisfying our analytic assumptions, a four-homogeneous term $b_{4}(\omega)$ would appear in (2.5); remarkably, it is exactly the restriction imposed on $U_{2}[A]$ by our conformal assumption that forces it to vanish and gives us a chance to have a convex $F(\omega)$. In higher even dimension $n$, terms of homogeneity up to $n$ would appear for general $A$ [B2]; it would be interesting to know whether the conformal assumption also forces the homogeneity $n$ term to vanish in this setting.

d. Higher powers of $\nabla^{2}$ (or of $D$ ) do not provide independent $\left(\beta_{1}, \beta_{2}, \beta_{3}\right.$ ) [FG]. But there are several other examples of conformal covariants which fit into our framework [B1, Pa, B2, B4]. Perhaps most accessible is the Paneitz operator $P$, the heat invariants of which are computable from [G2]. Among the conformal differential form Laplacians of [B1] is an operator $B$ on the middle $(n / 2)$ forms in even dimensions which has the same leading symbol as $\delta d-d \delta$. This implies that $B^{2}$ has leading term $\Delta^{2}$, and in fact, $B^{2}$ is an operator of the form treated in [G2]. [B4] gives a complete classification of second-order conformal covariants on tensor-spinor bundles. Though these operators almost never have metric leading symbol, many have positive definite leading symbol. It remains to develop a reasonable scheme for computing the heat invariants of operators whose leading symbol is not a power of the metric tensor.

e. Our explicit determinant formulas, are, of course, really formulas for quotients $(\operatorname{det} \bar{A}) /(\operatorname{det} A)$, where $A$ is computed in the very symmetric background metric $g$, and $\bar{A}$ is computed in the conformal metric $\bar{g}=e^{2 \omega} g$. It is a matter of some difficult but straightforward arithmetic to compute the denominators $\operatorname{det} A$. The necessary techniques are exactly those already employed in the twodimensional case [Polc, We1-2]. We have carried out extensive calculations in the case of the sphere $S^{4}$. Here, remarkably, the zeta functions for all of the conformally covariant operators mentioned above are built from the special zeta functions

$$
\begin{aligned}
& h_{a}(s)=\sum_{j=0}^{\infty}[(j+a)(j+a+1)]^{-s}, \\
& f_{a}(s)=\sum_{j=0}^{\infty}(2 j+2 a+1)[(j+a)(j+a+1)]^{-s},
\end{aligned}
$$

where $a$ is a positive real number, and from the classical Hurwitz zeta functions $\zeta_{a}(s)=\Sigma(j+a)^{-s}$. (Our explicit spectral computations in the standard metric 
are based on the representation theory of the rotation and spin groups $[\mathrm{B} 4, \S 5$; B5].) The simplest example is the conformal Laplacian, $\Delta+2$ in the standard metric. The eigenvalues of $\Delta+2$ are the $(j+1)(j+2)$ with multiplicities $(j+1)(j+2)(2 j+3) / 6, j=0,1,2, \ldots ;$ thus

$$
\zeta_{\Delta+2}(s)=\frac{1}{6} f_{1}(s-1) \text {. }
$$

A computation analogous to that in [We1, Appendix C] gives

$$
\operatorname{det}(\Delta+2)=\exp \left(-\frac{1}{144}-\frac{2}{3} \zeta_{R}^{\prime}(-3)-\frac{1}{3} \zeta_{R}^{\prime}(-1)\right)=1.04562 \ldots,
$$

where $\zeta_{R}(s)=\zeta_{1}(s)$ is the classical Riemann zeta function.

f. We expect the determinant to be crucial in the study of isospectral sets of metrics within a conformal class in dimension 4; partial results (indicating that such sets are "small" in an appropriate sense) have already been obtained [BCY]. The analogous problem in three dimensions was handled in [BPY, CY1-2]. In odd dimensions, the determinant is much more rigid under conformal deformation (the $U_{n / 2}$ term in (1.2) vanishes); as a result, the use of the determinant is an important qualitative difference between dimensions 3 and 4 . Note that the scale-invariant functionals treated in this paper are, like the determinant, spectral invariants: besides the determinant, they involve the volume (essentially $\left.\int U_{0}\right)$ and the trace anomaly $\zeta_{A}(0)$.

g. Conformal variational formulas for the heat invariants and determinant of a boundary-value problem, and for the eta function and eta invariant of a firstorder operator [BØ3, BG1-2] make it possible to use the methods of this paper to compute the eta invariant in three dimensions, and determinants of boundaryvalue problems in three and four dimensions. Formulas for these spectral invariants are potentially valuable in the study of corresponding uniformization and isospectral problems.

\section{REFERENCES}

[B1] T. Branson, Conformally covariant equations on differential forms, Comm. Partial Differential Equations 7 (1982), 392-431.

[B2] Differential operators canonically associated to a conformal structure, Math. Scand. 57 (1985), 293-345.

[B3] - Group representations arising from Lorentz conformal geometry, J. Funct. Anal. 74 (1987), 199-291.

[B4] _ Second-order conformal covariants, preprint.

[B5] Harmonic analysis in vector bundles associated to the rotation and spin groups, J. Funct. Anal. (to appear).

[BCY] T. Branson, S.-Y. A. Chang, and P. Yang, Estimates and extremal problems for the zeta function determinant on four-manifolds, preprint.

[BG1] T. Branson and P. Gilkey, The asymptotics of the Laplacian on a manifold with boundary, Comm. Partial Differential Equations 15 (1990), 245-272.

[BG2] _ Residues of the eta function for an operator of Dirac type, preprint. 
[BØ1] T. Branson and B. Ørsted, Conformal indices of Riemannian manifolds, Compositio Math. 60 (1986), 261-293.

[BØ2] - Conformal deformation and the heat operator, Indiana Univ. Math. J. 37 (1988), 83-110.

[BØ3] - Conformal geometry and global invariants, Differential Geometry and Applications (to appear).

[BPY] R. Brooks, P. Perry, and P. Yang, Isospectral sets of conformally equivalent metrics, Duke Math. J. 58 (1989), 131-150.

[CY1] S.-Y. A. Chang and P. Yang, Compactness of isospectral conformal metrics on $S^{3}$, Comment. Math. Helv. 64 (1989), 363-374.

[CY2] - Isospectral conformal metrics on 3-manifolds, J. Amer. Math. Soc. 3 (1990), 117-145.

[ES] M. Eastwood and M. Singer, A conformally invariant Maxwell gauge, Phys. Lett. 107A (1985), 73-74.

[FG] H. D. Fegan and P. Gilkey, Invariants of the heat equation, Pacific J. Math. 117 (1985), 233-254.

[G1] P. Gilkey, Spectral geometry of a Riemannian manifold, J. Differential Geometry 10 (1975), 601-618.

[G2] _ The spectral geometry of the higher order Laplacian, Duke Math. J. 47 (1980), 511-528.

[G3] - Invariance theory, the heat equation, and the Atiyah-Singer index theorem, Publish or Perish, Wilmington, Delaware, 1984.

[H] S. W. Hawking, Zeta function regularization of path integrals in curved spacetime, Comm. Math. Phys. 55 (1977), 133-148.

[K] Y. Kosmann, Sur les degrés conformes des opérateurs différentiels, C. R. Acad. Sci. Paris Ser. A 280 (1975), 229-232.

[OPS] B. Osgood, R. Phillips, and P. Sarnak, Extremals of determinants of Laplacians, J. Funct. Anal. 80 (1988), 148-211.

[Pa] S. Paneitz, A quartic conformally covariant differential operator for arbitrary pseudoRiemannian manifolds, preprint.

[Polc] J. Polchinski, Evaluation of the one loop string path integral, Comm. Math. Phys. 104 (1986), 37-47.

[Poly1] A. Polyakov, Quantum geometry of Bosonic strings, Phys. Lett. B 103 (1981), 207-210.

[Poly2] _ Quantum geometry of Fermionic strings, Phys. Lett. B 103 (1981), 211-213.

[Sc] R. Schoen, Conformal deformation of a Riemannian metric to constant scalar curvature, J. Differential Geometry 20 (1984), 479-495.

[Se] R. Seeley, Complex powers of an elliptic operator, Proc. Sympos. Pure Math. 10 (1967), 288-307.

[We1] W. Weisberger, Normalization of the path integral measure and the coupling constants for bosonic strings, Nuclear Physics B 284 (1987), 171-200.

[We2] - Conformal invariants for determinants of Laplacians on Riemann surfaces, Comm. Math. Phys. 112 (1987), 633-638.

Mathematical Institute, University of Copenhagen, Universitetsparken 5, 2100 Copenhagen, Denmark 52242

Current address: Department of Mathematics, University of Iowa, Iowa City, Iowa

Department of Mathematics and Computer Science, Odense University, Campusvej 55, 5230 Odense M, Denmark 